Bydgoszcz.

\title{
FRÄULEIN ELSES BEGEHREN: KOLORIERUNGEN VON EMOTIONEN IN ARTHUR SCHNITZLERS NOVELLE UND IN MANUELE FIORS COMIC
}

\begin{abstract}
Fräulein Else's Desire: Colorations of Emotions in the Novella by Arthur Schnitzler and the Graphic Novel by Manuele Fior

The following article compares the representation of nakedness in the novella Fräulein Else (1924) by Arthur Schnitzler and in the Graphic Novel Fräulein Else (2009) by Manuele Fior. While Schnitzler is inserting parts of the musical score Carnaval by Robert Schumann at that point of the text, when Fräulein Else is showing herself naked publically, and thus refuses to give more details, Fior cites in his Comic adaption the famous painting Nuda Veritas by Gustav Klimt, which was scandalized at its time. Slight changes are made also at the ending: while Schnitzler refers to the Orpheus-Eurydike-Myth, Fior compares the interior monologue of Fräulein Else to the myth of Echo.
\end{abstract}

KEYwORDS: Arthur Schnitzler, Fräulein Else, Nakedness, Music, Manuele Fior, Comic

Im Zentrum von Arthur Schnitzlers Novelle Fräulein Else (1924) steht ein Skandal: Eine 19-jährige junge Frau aus gehobenem Wiener Bürgertum entkleidet sich im Musiksalon ihres Ferienhotels vor aller Öffentlichkeit. Nur in einen schwarzen Mantel gehüllt, betritt sie den öffentlichen Musiksaal, in dem Schumanns Carnaval auf dem Klavier gespielt wird. Plötzlich reißt sie ihren Mantel auf, lässt ihn fallen und steht nackt vor den Hotelgästen, um im nächsten Augenblick ohnmächtig zu Boden zu fallen.

Mit der Nacktszene greift Schnitzler ein Motiv auf, das um die Jahrhundertwende zu einem zentralen Thema avanciert. Während schamlose Frauen wie Salomé, Carmen und Lulu zu Heldinnen in literarischen Texten werden und auf Gemälden von Gustav Klimt und Egon Schiele lasziv entblößte Körper zu sehen sind, zeigen sich Ausdruckstänzerinnen auf den Varietébühnen der großen europäischen Metropolen erstmals unverhüllt. Mit ihren Darstellungen provozieren die Kultur- 
schaffenden nicht selten Skandale, für die sie sich vor Gericht verantworten müssen. Zugleich fungieren sie als Seismographen einer sich drastisch verändernden Zeit, in der insbesondere der weibliche Körper zunehmend in die Öffentlichkeit tritt. Durch das Ablegen des Korsetts, das Aufkommen neuer Kleidermoden und Tanzstile, aber auch durch die Freikörperbewegung und die neuen Reproduktionsmedien Fotografie und Film verändert sich die Wahrnehmung von Körpern nachhaltig.

Gab der weibliche Nacktauftritt im Fin de Siècle noch Anlass zum Skandal, wird dieser in den Wilden Zwanzigern als weniger anstößig erachtet. Es haben sich feste Räume etabliert, in denen der Nacktauftritt nicht nur erlaubt, sondern geradezu als Spektakel aufgeführt wird. Da Fräulein Else keine Nackttänzerin ist und ihr Auftrittsort auch kein Varieté der 1920er Jahre, sondern der Musiksalon eines Grand Hotels um die Jahrhundertwende, in dem die gehobene bürgerliche Gesellschaft verkehrt, verstößt ihre öffentliche Entblößung gegen die üblichen Konventionen: Elses Auftritt wird als schamlos empfunden. Er fällt aus dem Rahmen und kann als transgressive „Geste der Vergeudung und Überschreitung “" verstanden werden, die Schnitzler eng mit der Frage nach der Darstellbarkeit verbindet. So zeigt und verhüllt der Text gleichermaßen das Skandalon, indem er - ohne den Moment der Enthüllung detailliert zu beschreiben - etwas anderes (nämlich Noten) an seine Stelle setzt und dadurch indirekt darauf verweist. Der Nacktauftritt wird so geschickt dem voyeuristischen Begehren der Leser/innen entzogen, dafür werden jedoch die Grenzen der Darstellung ausgelotet und die ,Schamlosigkeit der Undarstellbarkeit" gezeigt.

Fehlten in der ersten Fassung von Fräulein Else, die in der Neuen Rundschau im Oktober 1924 als Vorabdruck veröffentlicht wurde, noch die drei Auszüge aus der Notenschrift von Robert Schumanns Carnaval, arbeitet Schnitzler den Darstellungsentzug in der zweiten endgültigen Textfassung, die am 25. November 1924 im Zsolnay-Verlag erscheint, durch die entscheidende Einfügung noch markanter heraus. ${ }^{2}$ An dem Punkt des Texts, wo die Sprache versagt, greift Schnitzler auf ein anderes Notationssystem (die Musik) zurück und markiert auf diese Weise das Versagen des Textes.

Insbesondere durch die bewusste Verwendung von Satzzeichen und Auslassungen arbeitet Schnitzler die Darstellungsproblematik und die Sprachskepsis, wie sie typisch für die Wiener Moderne sind, in der gesamten Novelle durch. Auch die

\footnotetext{
${ }^{1}$ Gabriele Brandstetter: Divested Interests. Ökonomie der Entblößung in Arthur Schnitzlers „Fräulein Else “ und Marina Abramovic' ,Freeing the Body“. In: Kerstin Gernig (Hrsg.): Nacktheit. Ästhetische Inszenierungen im Kulturvergleich. Köln/Weimar/Wien 2002, S. 241-271, hier S. 242.

${ }^{2}$ Die Buchausgabe der Novelle erschien anfangs nicht bei S. Fischer, sondern in Wien beim Paul Zsolnay Verlag. Bis zur Verfilmung im Jahr 1929 wurden 70.000 Exemplare gedruckt und verkauft. 1928 nahm Arthur Schnitzler die Novelle in den 6. Band seiner bei S. Fischer publizierten Gesammelten Werke auf. Zu weiteren Fräulein-Else-Ausgaben vgl. Reinhard Urbach: Schnitzler-Kommentar zu den erzählenden Schriften und dramatischen Werken. München 1974, S. 130-132, hier S. 130.
} 
exzessive Benutzung von Wortspielen, geborgten Worten und abgegriffenen Sprachklischees, die darüber hinaus als solche gleich mehrfach im Text zum Thema gemacht werden, tragen ebenso dazu bei wie die komplexe Erzählsituation des inneren Monologs.

\section{Elses unerhörtes Kopftheater}

Es ist der innere Monolog - die „unmittelbare Aeußerung“ der Titelfigur, das „Denken, Fühlen und Sprechen genau in der Sekunde des Erlebens“ ${ }^{\text {“3 }}$ - der eine Erzählsituation schafft, in der Selbstoffenbarung und (Ver-)Schweigen, Ent- und Verhüllen, Erkennen und Verkennen gleichzeitig möglich sind. Der Text inszeniert dadurch einen Seelenstriptease, der nicht nur Aufschluss über Fräulein Elses Innenleben und Begehren gibt, sondern auch gesellschaftliche Machtstrukturen offenlegt.

Schon damals ist Elses Rede ,als sensibles und mitfühlendes Psychogramm einer jungen Frauengeneration“4 gelesen worden, der Schnitzler eine - wenn auch im Text unhörbar bleibende - so doch für die Leser/innen deutlich vernehmbare Stimme verliehen hat. Else fungiert als Sprachrohr einer neu aufkommenden Frauengeneration, die sich - trotz Aufbruch und entscheidenden Veränderungen - häufig noch in einem ausweglosen Dilemma befindet. Zwischen Selbstermächtigung und Ohnmacht, Gehör einfordernd und in Aphonie verfallend bewegen sich diese Frauen in einer paradoxen Situation.

Vor allem die literarische Konstruktion des inneren Monologs erweist sich als ideal, um Elses Zwiespalt zwischen ausgeprägtem Ausdruckswillen und sozialer Stummheit, Selbstermächtigungsanspruch und Ohnmacht adäquat wiedergeben zu können. Wie schon bei Leutnant Gustl (1900) nutzt Schnitzler den inneren Monolog und konstituiert dadurch Else als Ich-Erzählerin und Selbst-Darstellerin. „An die Stelle des Leutnants, der männlichen Leitfigur der Jahrhundertwendegesellschaft, rückt [...] eine junge Frau, die ihren Ort in dieser Gesellschaft noch finden muss. ${ }^{.5}$ Jung, schön, sportlich und klug macht Else mit ihrer Tante und ihrem Cousin Paul Urlaub in den italienischen Dolomiten, wo ein Expressbrief der Mutter sie in eine außergewöhnliche Situation versetzt: Denn Else soll die Familie, die aufgrund der Spielleidenschaft des Vaters kurz vor dem Bankrott steht, retten, „indem sie von

\footnotetext{
${ }^{3}$ Felix Salten: „Fräulein Else“. In: Neue Freie Presse. Morgenblatt. Nr. 21623. Wien, Sonntag, den 23. November 1924, Titelseite bis S. 3. Vgl. auch die Rezension, die in Auszügen abgedruckt worden ist bei Evelyne Polt-Heinzl: Erläuterungen und Dokumente. Arthur Schnitzler „Fräulein Else“. Stuttgart 2002, S. 54-55.

${ }^{4}$ Konstanze Fliedl: Arthur Schnitzler. Stuttgart 2005, S. 217.

${ }^{5}$ Michael Scheffel: Nachwort. In: Arthur Schnitzler: Spiel im Morgengrauen. Erzählungen 1923-1931. Mit einem Nachwort von Michael Scheffel. Ausgewählte Werke in acht Bänden. Hrsg. von Heinz Ludwig Arnold. Bd. 3. Frankfurt a. M. 1999, S. 385-394, hier S. 387.
} 
einem zufällig im gleichen Hotel wohnenden Geschäftsfreund schnellstmöglich eine große Geldsumme beschafft. "60 Diese ausweglose Lage ruft bei Else wild-assoziative Gedanken über das eigene Selbstverständnis, aber auch über die generellen Erwartungen der Gesellschaft an eine bürgerliche Frau bzw. Tochter hervor.

Bezeichnend für den inneren Monolog ist, dass weniger Gefühle in der ersten Person ausgedrückt werden als ,dass eine innere Sprache lesbar gemacht wird“"7 , die normalerweise nicht zum Ausdruck kommt. Als „Experiment mit dem Unausgesprochenen“ ${ }^{\text {8 }}$ ist der innere Monolog „Form gewordene Introversion““9 . Er ermöglicht dem Leser, zum Mithörer von aphoner Sprache zu werden, zum „Voyeur, der die Gedanken einer Person ohne deren Billigung liest. “10 Der Leser des inneren Monologs gleicht damit einem Gedankenleser, wobei die stumme Unmittelbarkeit weder editiert noch einer Revision unterzogen worden zu sein scheint.

Unmittelbar, unrein und unediert wirkt auch der Wortfluss, den Else produziert. Die Leser werden mit einem ungeordneten, wirren und sprunghaften Kopftheater konfrontiert, das auch auf der formalästhetischen Ebene des Textes sichtbar wird. Von einer Assoziation springt Else zur anderen und bewirkt dadurch die „Atomisierung des Erzählten in Bewusstseinspartikel“"11. Vereinzelt entstehen dadurch auch doppelsinnige Wortspiele, die Zeugnis von Elses Sprachwitz und Klugheit ablegen.

In ihren Gedanken und Tagträumen nimmt Else spielerisch verschiedene Rollen ein, erprobt und verwirft sie wieder, schwankt ,zwischen Wunschträumen und klarstem Realitätssinn.“12 „Ich“ heißt für sie „,die Hochgemute, die Aristokratin, die Marchesa, die Bettlerin, die Tochter des Defraudanten“ $(18)^{13}$ sowie das ,sporting girl“ (18). Sie ist alles und nichts, ein Chamäleon, das je nach Situation und Bedarf sich verändert, andere Posen einnimmt und in unterschiedliche Frauenrollen schlüpft.

In ihrem Gedankenspektrum spielt Else insbesondere die Möglichkeiten durch, die vielen bürgerlichen Töchtern nach dem Ersten Weltkrieg - nach Inflation und Börsencrash - tatsächlich als einzige Optionen bleiben: nämlich entweder reich zu

${ }^{6}$ Ebd.

${ }^{7}$ Mario Gomes: Gedankenlesemaschinen. Modelle für eine Poetologie des Inneren Monologs. Freiburg i.Br./Berlin/Wien 2008, S. 120.

${ }^{8}$ Craig Morris: Der vollständige innere Monolog: eine erzählerlose Erzählung? Eine Untersuchung am Beispiel von ,Leutnant Gustl“ und „Fräulein Else“. In: Modern Austrian Literature. Journal of the International Arthur Schnitzler Research Association 31, Nr. 2 (1998), S. 30-51, hier S. 44.

${ }^{9}$ Erika Hoehnisch: Das gefangene Ich. Studien zum inneren Monolog in modernen französischen Romanen. Heidelberg 1967, S. 122.

${ }^{10}$ Gomes: Gedankenlesemaschinen, S. 74.

${ }^{11}$ Diersch: Empiriokritizismus und Impressionismus, S. 97.

${ }^{12}$ Jürgen Zenke: Die deutsche Monologerzählung im 20. Jahrhundert. Köln/Wien 1976, S. 62.

${ }^{13}$ Arthur Schnitzler: Fräulein Else. In: ders.: Spiel im Morgengrauen. Erzählungen 1923-1931. Mit einem Nachwort von Michael Scheffel. Ausgewählte Werke in acht Bänden. Hrsg. von Heinz Ludwig Arnold. Bd. 3. Frankfurt a. M. 1999, S. 7-73. Im Folgenden werden die Zitate in Klammern direkt im Haupttext nachgewiesen. 
heiraten und ein tristes Leben als Ehefrau eines häufig älteren Ehemanns zu führen und damit ihr gerade erst aufgeflammtes Begehren endgültig einzufrieren (ähnlich wie Effie Briest, Madame Bovary oder auch Anna Karenina dies in den Romanen der Jahrhundertwende tun) oder als Bonne, Telefonistin oder Ladenmädchen zu arbeiten und damit in eine prekäre Lebenssituation zu geraten, stets am Rande von Armut und Prostitution, wo das eigene Begehren ebenfalls nicht ausgelebt werden kann, sondern höchstens lauwarm bleibt. ${ }^{14}$ Dass ihre eigenen Wünsche anders aussehen und dass sie sich einen anderen Lebensweg für sich erträumt, wird immer dann deutlich, wenn Else sich als schillernde, heißblütige Femme fatale imaginiert, die auf kalten Marmorstufen in der Sonne liegend nicht nur unendlich begehrenswert erscheint, sondern vielmehr selbst leidenschaftlich begehrt.

Doch das Spielerische, das Elses Gedanken- und Rollenspielen am Anfang ansatzweise noch eignet, verblasst spätestens dann, als sie den Expressbrief von ihrer Mutter gelesen hat. Die Mutter bittet sie darin, den Kunsthändler Herrn von Dorsday, den der Vater schon einmal juristisch erfolgreich vertreten hat und der im gleichen Hotel wie Else Urlaub macht, zu fragen, ob er der Familie ,aushelfen' kann. Else ahnt sofort, welche Art von Handel damit verbunden ist. Nur schwer ringt sie sich dazu durch, Dorsday als Bittstellerin aufzusuchen. Als sie ihn schließlich aufsucht, ist die Situation „von Anfang an als ein sexuelles Machtspiel grundiert.“" ${ }^{15}$ Dorsdays Gegenforderung für das ,Aushelfen' lässt so auch nicht lange auf sich warten.

Sein Verlangen, Else eine Viertelstunde nackt sehen zu wollen, kann sogar als Kontrapunkt zu den vielen unterschiedlichen fragmentarischen Weiblichkeitsbildern, die Else in der Novelle zeitweise erprobt, gedeutet werden. Denn nackt, stillgestellt, fest gerahmt und ganz, wünscht Dorsday Else zu sehen. Es geht um „die Inszenierung einer Idealisierung “ ${ }^{\star 16}$, die Dorsday aus sicherer Distanz genießen möchte.

Um Dorsdays unangemessener Forderung standhalten zu können, entscheidet sich Else nach langem Ringen für eine Selbstentblößung, die seine Bedingung zwar einerseits erfüllt, sie andererseits aber auch unterläuft. Die Raffinesse von Elses Überlegung, sich in der Öffentlichkeit zu entblößen, besteht darin, wie Brigitte Prutti bemerkt,

dass das zum privaten Genuss vorgesehene weibliche Objekt den Schein der Intimität durchbricht und mit dem Skandalon ihrer öffentlichen Enthüllung auch den voyeuristischen männlichen Blick entlarvt, der ja der Hülle bedarf, um über die Schönheit des jungfräulichen Körpers phantasieren zu können ${ }^{17}$.

\footnotetext{
${ }^{14}$ Vgl. dazu Wendelin Schmidt-Dengler: Inflation der Werte und Gefühle. Zu Arthur Schnitzlers „Fräulein Else“. In: Giuseppe Farese (Hrsg.): Akten des Internationalen Symposiums „Arthur Schnitzler und seine Zeit". Bern 1985, S. 170-181, hier S. 175.

${ }^{15}$ Brandstetter: Divested Interests, S. 250.

${ }^{16}$ Ebd.

${ }^{17}$ Brigitte Prutti: Weibliche Subjektivität und das Versagen des sanften Patriarchen in Schnitzlers „,Fräulein Else“. In: Orbis Litterarum Nr. 59 (2004), S. 159-187, hier S. 172.
} 
Indem Else Dorsday um seinen exklusiven, im Dunklen verbleibenden Blick bringt, lenkt sie geschickt von sich ab und lässt ihn, aber auch die anderen ,Herrschaften“ der bürgerlichen Gesellschaft, in ihrem voyeuristischen Begehren sichtbar werden. Die Selbstentblößung wird zur Bloßstellung der Gesellschaft und ihrer Doppelmoral. Der Rahmen wird damit weiter gefasst als ursprünglich vorgesehen: Nicht nur Else wird vom Rahmen erfasst, sondern auch die Rahmenbedingungen und ihre genderspezifischen Implikationen rücken in den Blick.

komm! Sein Auge spricht: ich will dich nackt sehen. Nun, du Schuft, ich bin ja nackt. Was willst du denn noch? Schick die Depesche ab... Sofort... Es rieselt durch meine Haut. Die Dame spielt weiter.
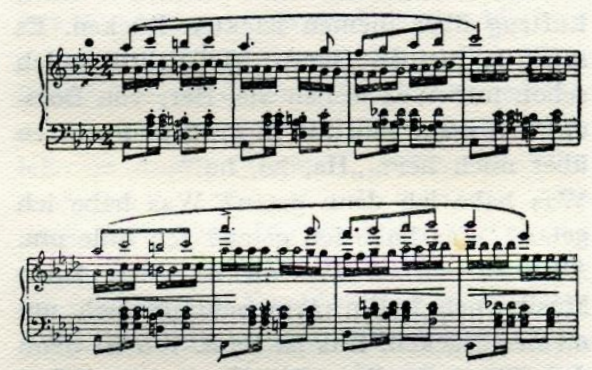

Köstlich rieselt es durch meine Haut. Wie wundervoll ist es nackt zu sein. Die Dame spielt weiter, sie weiß nicht, was hier geschieht. Niemand weiß es. Keiner noch sieht mich. Filou, Filou! Nackt stehe ich da. Dorsday reißt die Augen auf. Jetzt endlich glaubt er es. Der Filou steht auf. Seine Augen leuchten. Du verstehst mich, schöner Jüngiing. „Haha!“ Die Dame spielt nicht mehr. Der Papa ist gerettet. Fünfzigtausend! Adresse bleibt Fiala! „Ha, ha, ha!“ Wer

\section{7}

Abb. 1. Erstausgabe: Arthur Schnitzler: Fräulein Else. Novelle. Berlin/Wien/Leipzig: Paul Zsolnay 1924

Abgesehen davon weigert sich Else mit ihrer persönlichen Vorstellung auch, überkommene Bildklischees des 19. Jahrhunderts nachzustellen. So gefriert sie weder zu einer lebenden Marmorstatue noch fügt sie sich harmonisch in die Landschaft ein oder liegt verführerisch auf einem Diwan im Interieur - wie von Dorsday als 
mögliche Optionen vorgeschlagen. Stattdessen kreiert Else ein Selbstbild, für das sie die alleinige Autorschaft beanspruchen kann und das in seiner plötzlichen Heftigkeit, Frontalität und Unmittelbarkeit nachhaltig verstört. Statt einer , begehrenswerten' Inszenierung (beispielsweise durch das Einnehmen einer lasziven Pose) bietet sie Herrn Dorsday (und damit auch den Leser/innen) eine ,kalte', analytische Präsentation der Problematik des weiblichen Begehrens.

Dass es Schnitzler genau darum geht, wird auch auf der formalästhetischen Ebene des Textes deutlich. So entzieht sich die Nacktszene buchstäblich der Repräsentation. Nur durch ein „hier“ (63) sowie durch einen Auszug aus der Notenpartitur von Robert Schumanns Carnaval, ${ }^{18}$ die quasi als Platzhalter für das Undarstellbare fungiert, wird deiktisch auf die Selbstentblößung von Else hingewiesen (Abb. 1). Indem ihr nackter Körper in der Novelle nicht beschrieben, sondern auf ihn nur verwiesen wird, hinterlässt er eine Leerstelle im Text. Enthüllt wird er sogleich wieder verhüllt.

„Nackt stehe ich da“ (63) - mehr ist in der entsprechenden Stelle des Textes nicht zu lesen. Die Leser/innen werden - ähnlich wie Dorsday - mit einem Moment des Entzugs konfrontiert und dadurch um ihr voyeuristisches Begehren gebracht. Statt mit detaillierten ,nackten Tatsachen' über die Art und Weise der Entblößung werden die Leser/innen mit Elses Stimme des Begehrens konfrontiert.

\section{Elses Stimme des Begehrens}

Als Unterbrechung des Schriftbildes verweisen die Notenauszüge von Robert Schumanns Carnaval ganz besonders auf das Unaussprechliche, das sich an dieser Stelle der Novelle zeigt. Statt auf Worte greift Schnitzler auf die emotionale Sprache von Musik zurück, um „die äußerst subtilen seelischen Vorgänge eindringlicher und zarter [zu] beschreiben, als eine Schilderung durch Worte es vermocht hätte. “19 So wird beispielsweise das Stürmische von Elses Charakter durch einen häufigen Wechsel der Tonstärken in den ersten zwei musikalischen Bruchstücken suggeriert,

${ }^{18}$ Das Einfügen von optischen Musikzitaten ist 1924 keine Neuheit mehr, wie Martin Huber konstatiert: „Durch Balzacs Modeste Mignon (1844) und ganz besonders durch Romain Rollands Bestseller Jean-Christoph (1904-12) dürfte dieses Verfahren den Lesern durchaus geläufig gewesen sein.“ (Martin Huber: Optische Musikzitate als Psychogramm in Arthur Schnitzlers „Fräulein Else“. In: ders.: Text und Musik. Musikalische Zeichen im narrativen und ideologischen Funktionszusammenhang ausgewählter Erzähltexte des 20. Jahrhunderts. Frankfurt a. M./Berlin/Bern/New York/Paris/Wien 1991, S. 78-91, hier S. 79). Haben die Notenzitate in diesen Texten ausschließlich einen illustrierenden und atmosphärischen Charakter, kommt ihnen bei Schnitzler eine weit komplexere Funktion zu.

${ }^{19}$ Gerd K. Schneider: Ton- und Schriftsprache in Schnitzlers „Fräulein Else“ und Schumanns „Carnaval“. In: Modern Austrian Literature. Journal of the International Arthur Schnitzler Research Association. Vol. 2, Nr. 3 (Winter 1969), S. 17-20, hier S. 17. 
wobei dieser unmittelbar „,zu der inneren Dynamik Elses, dem Anschwellen und Abnehmen der Intensität ihrer Gefühlswelt in Beziehung gesetzt werden “20 kann (Abb. 2). Heide Eilert spricht deshalb auch von einer opernhaften Überhöhung von Elses , großer Vorstellung' durch die Musik. ${ }^{21}$

das Spielzimmer. Grüner Vorhang vor der Tür. Man sieht nichts. Ich stelle mich auf die Zehenspitzen. Die Whistpartie. Die spielen jeden Abend. Dort spielen zwei Herren Schach. Herr von Dorsday ist nicht da. Viktoria. Gerettet! Wieso denn? Ich muß weiter suchen. Ich bin verdammt, Herrn von Dorsday zu suchen bis an mein Lebensende. Er sucht mich gewiß auch. Wir verfehlen uns immerfort. Vielleicht sucht er mich oben. Wir werden uns auf der Stiege treffen. Die Holländer sehen mich wieder an. Ganz hübsch die Tochter. Der alte Herr hat eine Brille, eine Brille, eine Brille... Fünfzigtausend. Es ist ja nicht so viel. Fünfzigtausend, Herr von Dorsday. Schumann? Ja, Karneval ... Hab' ich auch einmal studiert. Schön spielt sie.

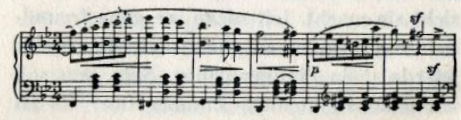

Warum denn sie? Vielleicht ist es ein Er? Vielleicht ist es eine Virtuosin? Ich will einen Blick in den Musiksalon tun.

Da ist ja die Tür. - - Dorsday! Ich falle um. Dorsday! Dort steht er am Fenster und hört zu. Wie ist das möglich? Ich verzehre mich - ich werde verrückt - ich bin tot und er hört einer fremden Dame Klavierspielen zu. Dort auf dem Divan sitzen zwei Herren. Der Blonde ist erst heute angekommen. Ich hab' ihn aus dem Wagen steigen sehen. Die Dame ist gar nicht mehr jung. Sie ist schon ein paar Tage lang hier. Ich habe nicht gewußt, daß sie so schön Klavier spielt. Sie hat es gut. Alle Menschen

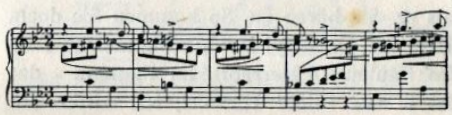

haben es gut ... nur ich bin verdammt... Dorsday! Dorsday! Ist er das wirklich? Er sieht mich nicht. Jetzt schaut er aus, wie ein anständiger Mensch. Er hört zu. Fünfzigtausend! Jetzt oder nie. Leise die Tür aufgemacht. Da bin ich, Herr von Dorsday! Er sieht mich nicht. Ich will ihm nur ein Zeichen mit den Augen geben, dann werde ich den Mantel ein wenig lüften, das ist genug. Ich bin ja ein junges Mädchen. Bin ein anständiges junges Mädchen aus guter

115

Abb. 2. Erstausgabe: Arthur Schnitzler: Fräulein Else. Novelle. Berlin/Wien/Leipzig: Paul Zsolnay 1924

Bezeichnend ist darüber hinaus auch der Gegensatz, der in der Selbstenthüllungsszene zwischen Fräulein Else und der Klavierspielerin aufgemacht wird. Elses Vorstellung steht nämlich im deutlichen Kontrast zum Vorspiel der Pianistin, der Dorsday andächtig zuhört, als Else den Musiksaal betritt. War es um die Jahrhundertwende gemeinhin üblich, dass die bürgerlichen Töchter Klavier spielen lernten, um vor kleinerem Publikum im Salon zu reüssieren und dadurch eventuell zukünftige Ehemänner zu beeindrucken, die die Frauen während ihrer Darbietung in Ruhe

${ }^{20}$ Ebd.

${ }^{21}$ Vgl. Heide Eilert: Das Kunstzitat in der erzählenden Dichtung. Studien zur Literatur um 1900. Stuttgart 1991, S. 323-335, hier S. 331. 
betrachten konnten, wechselt Else das Genre ihres Virtuosentums. Obwohl sie auch das Klavierspiel beherrscht, zieht sie für ihre ,große Vorstellung' den (Nackt-)Tanz als persönliches Ausdrucksmittel vor. Keine stillgestellte Statue zeigt sie der Öffentlichkeit, sondern eine höchst bewegte Frau. Else ist deshalb auch kein artiste manqué, die Kunst nur reproduzieren kann, ,indem sie Klavier spielt oder sich pantomimisch zu Schumanns Carnaval bewegt ${ }^{\text {‘22 }}$, wie Astrid Lange-Kirchheim meint. Vielmehr tritt sie als radikale Bildzerstörerin auf, die den gesellschaftlichen Rahmen sprengt.

Die Funktion der Noten scheint deshalb weit darüber hinaus zu gehen, lediglich die Klaviatur von Elses Gefühlen darzustellen. Schließlich hat sich Schnitzler, der selbst leidenschaftlicher Konzertgänger war und bravourös Klavier spielen konnte, ${ }^{23}$ mit Schumanns Carnaval (1835 fertig gestellt und 1837 publiziert) für ein äußerst sperriges Musikstück entschieden. Denn Schumanns Vorliebe mit Buchstaben und Noten zu spielen und diese auf alle möglichen Arten und Weisen zu variieren, erinnert bereits an die serielle Musik des 20. Jahrhunderts. Carnaval macht es deshalb auch Zuhörern und Interpreten gleichermaßen schwer, das Werk als einheitliches wahrzunehmen und nicht nur die einzelnen Sätze, die einander auszulöschen scheinen, in ihrer Heterogenität zu sehen.

$\mathrm{Zu}$ Schumanns serieller Technik gehört auch, dass er Musikzitate von Beethoven über Schubert bis Chopin verwendet. Mit seinem musikalischen Montageverfahren nimmt er (post-)moderne Schreibtechniken vorweg, die auch an Schnitzlers ästhetische Verfahren in Fräulein Else erinnern. Indem Schnitzler Partituren von Schumann in seine Novelle einbaut, verweist er auf sein eigenes Montageverfahren und ,entblößt' den Text als einen montierten, der zudem gerade an dieser Stelle an seine sprachlichen Grenzen stößt. Inszeniert wird ein Versagen des Textes. Die Krisis der Protagonistin fällt mit einer Sprachkrise zusammen. Im Angesicht des ,Nackten“ kommt es zur Erfahrung eines Sprachverlusts: „Wo dem Verlangen nach rückhaltloser Enthüllung nachgegeben wird, stellen sich Bilder des Gestaltverlusts, der Auflö-

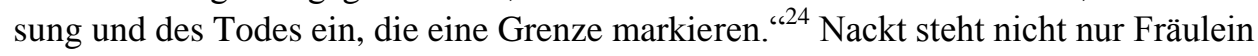
Else da, sondern auch die Sprachzeichen in ihrer bloßen Verweisfunktion.

Darüber hinaus markiert die Inserierung der Notenauszüge aber noch etwas anderes im Text, nämlich einen deutlichen Registerwechsel vom Visuellen zum Akustischen, vom Seh- zum Hörbaren. Denn wirklich Sinn machen die Auszüge der No-

${ }^{22}$ Astrid Lange-Kirchheim: Die Hysterikerin und ihr Autor. Arthur Schnitzlers Novelle „Fräulein Else“ im Kontext von Freuds Schriften zur Hysterie. In: Thomas Anz (Hrsg.): Psychoanalyse in der modernen Literatur. Kooperation und Konkurrenz. Würzburg 1999, S. 111-134, hier S. 129.

${ }^{23}$ Dass die Auswahl der Notenzitate nicht beliebig ist, zumal Schnitzler selbst Klavier spielte, ja sogar kleinere Stücke für dieses Instrument komponierte, hat Martin Huber in seiner Studie hervorgehoben. Vgl. Huber: Optische Musikzitate als Psychogramm in Arthur Schnitzlers ,,Fräulein Else“, S. 83.

${ }^{24}$ Caroline Pross: Näherungswerte. Theatralik des Wirklichen und realistisches Erzählen bei Arthur Schnitzler. In: Ethel Matala de Mazza, Clemens Pornschlegel (Hrsg.): Inszenierte Welt. Theatralität als Argument literarischer Texte. Freiburg i.Br. 2003, S. 227-249, hier S. 246. 
tenpartitur nur, wenn die Leser/innen sie nicht nur als Notenbild wahrnehmen, sondern auch im Kopf akustisch zum Klingen bringen. Der Registerwechsel lässt sich vor allem auf der inhaltlichen Ebene des Textes bemerken: Wird das visuelle Bild der nackten Else den Leser/innen in der Novelle entzogen, bekommen sie deren Stimme nach der Störung des Schriftbildes umso deutlicher zu hören. Die Stimme von Else, die im Gespräch mit anderen zuvor immer wieder brüchig, zittrig und leise zu werden drohte, verlangt am Ende der Novelle vehement danach, endlich Gehör $\mathrm{zu}$ finden. Auch wenn sie eigentlich schweigen soll, kann sie nicht anders als zu schreien, um Hilfe zu flehen und schließlich laut und deutlich in einen wunderschönen Chor einzustimmen, der einen „Augen-Blick des Hörens, eine Möglichkeit eines anderen, nicht an Bilder gebundenen Gedächtnisses ${ }^{\text {“25 }}$ aufblitzen lässt, wie Andrea Allerkamp konstatiert.

In der Forschung ist mehrfach darauf hingewiesen worden, dass sich in der Novelle deutliche Bezüge auf den Orpheus-und-Eurydike-Mythos finden lassen, der auf komplexe Weise Themen wie Wieder-sehen-wollen und Nicht-ansehen-dürfen, Blicken und Töten, Gehör-finden-wollen, Singen und (Be-)Klagen verbindet. Die Nymphe Eurydike, die von manchen auch Agriope (= ,die mit dem wilden Gesicht“) oder auch Argiope (= „,ie mit dem weißen Gesicht" $)^{26}$ genannt wurde, tritt eines Tages auf eine Schlange, als Aristaios sie zu vergewaltigen versucht, und stirbt. Ihr Mann Orpheus folgt ihr in den Hades, um sie auf seiner Leier spielend zu befreien. Der magische Klang seines Instruments erweicht dort alle. Auch Persephone ist von Orpheus musikalischer Wehklage dermaßen angetan, dass sie ihm erlaubt, seine Frau wieder mit sich hinaufzuführen. Allerdings nur unter der Bedingung, dass er sie dabei nicht anschauen darf. Da Orpheus Eurydike auf dem langen Weg aus dem Hades hinter sich plötzlich nicht mehr wahrnimmt, dreht er sich doch kurz zur Vergewisserung um, was allerdings ihren unmittelbaren Tod bedeutet. Orpheus Unfähigkeit, Eurydike ausschließlich akustisch wahrnehmen zu können, gepaart mit seiner Sehnsucht, sie (an-)sehen zu wollen, führen letztendlich zu ihrem endgültigen Tod.

Ähnlich wie Orpheus ergeht es den Leser/innen der Novelle. Auch ihnen reicht es nicht, ausschließlich Elses Stimme zu hören. Der Drang sie (nackt) zu sehen bzw. ein Bild von ihr vor dem inneren Auge der Immagination zu haben, ist viel zu groß. Taub für Elses zarte Kantilene des Begehrens können sie die Augen nicht von ihr lassen. Viel lieber als ihre Stimme (in Form des inneren Monologs) zu hören, möchten sie Else (nackt) sehen. Durch dieses Verlangen werden die Leser/innen zu Komplizen von Dorsday, Cissy, Paul, Fred, Papa und Mama, die gleichermaßen - wie Else es an einer Stelle der Novelle selbst bemerkt - ihren Tod mit zu verschulden haben.

${ }^{25}$ Andrea Allerkamp: „Ich schreibe ja keine Memoiren“. Über die ars memoriae als Spiel zwischen Bild und Text in Schnitzlers „Fräulein Else“. In: Cahiers d'Etudes Germaniques. Heft 29 (1995), S. 95-108, hier S. 108.

${ }^{26}$ Vgl. Karl Krényi: Die Mythologie der Griechen - Die Götter- und Menschheitsgeschichten. München 1994. 
Der Wunsch des Lesers nach restloser Enthüllung führt am Schluss der Novelle so auch buchstäblich zum Zerfallen des Textkorpus (Abb. 3). Die Sätze werden immer kürzer, bis schließlich einzelne Wörter gänzlich in sich zusammenbrechen. „Die Punkte im Schriftbild der Novelle sind die äußere Entsprechung dieser inneren Atomisierung. “27

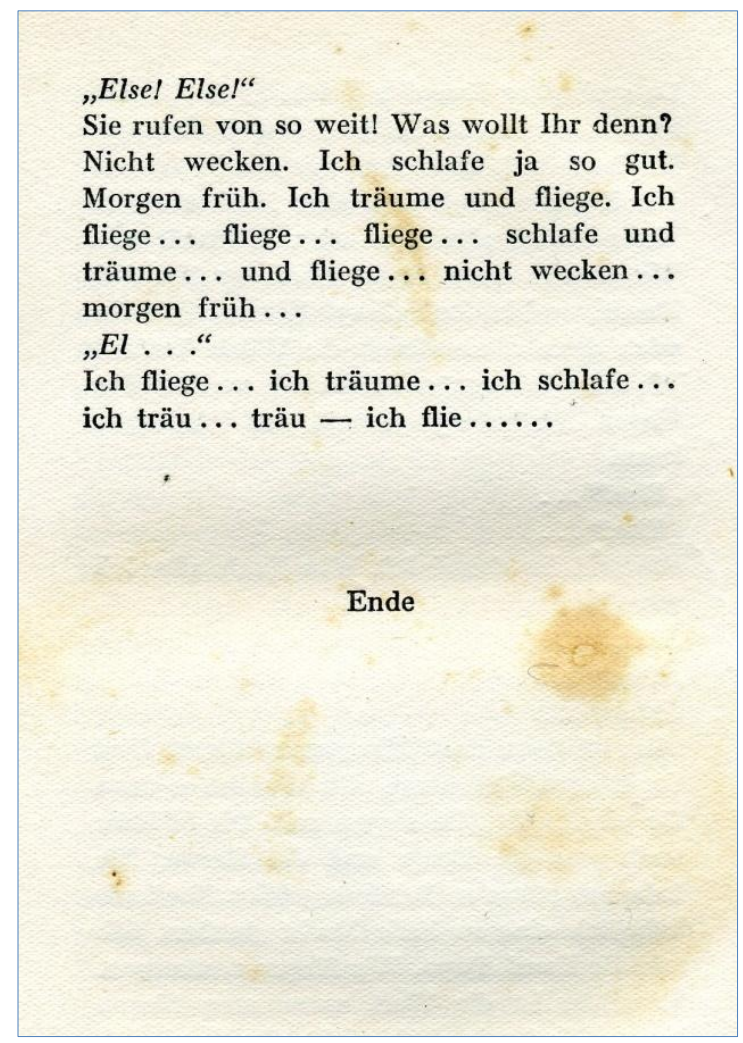

Abb. 3. Erstausgabe: Arthur Schnitzler: Fräulein Else. Novelle. Berlin/Wien/Leipzig: Paul Zsolnay 1924

Völlig entblößt liegt das Textkorpus selbst vor den Augen der Leser/innen. Schamlos werden das Schriftbild als solches und damit im Grunde auch die toten Sprachzeichen in ihrer bloßen Verweisfunktion enthüllt. Erschreckend ist, dass das unverhüllte Zeigen einer hüllenlosen Nacktheit bis zum Schluss widerständig bleibt und nichts wirklich preis gibt. Die letzte weiße Seite des Buches wird zu einem schneeweißen Totenlaken, unter dem Else den Leser/innen für immer verborgen bleiben wird. Flüstert der Text den Leser/innen zwar scheinbar permanent ELSE

\footnotetext{
${ }^{27}$ Diersch: Empiriokritizismus und Impressionismus, S. 99.
} 
LESE(N) (ein Annagramm von Else) zu, entpuppen sich die Leser/innen in ihrem Begehren nach endgültiger Enthüllung der Textbedeutung letztendlich als ESEL (auch ein Annagramm von Else).

Kurios ist, dass jener Text, der derart obsessiv um das Problem der Darstellung kreist und einen Bildentzug in Szene setzt, in den letzten Jahrzehnten vor allem zu visuellen Neuinterpretationen im Stummfilm, im Fernsehen und im Arthouse-Kino geführt hat. ${ }^{28}$ Waren es lange vornehmlich Verfilmungen, die literarische Klassiker durch die filmische Transponierung wieder populär gemacht haben, kommt diese Funktion heute zunehmend den Literatur-Comics zu. Es erstaunt deshalb nicht, dass auch Schnitzlers Novelle - im Zuge der zahlreichen Graphic Novels, die in den letzten Jahren entstanden sind - ebenfalls eine Comic-Adaption hervorgerufen hat, die abschließend näher betrachtet werden soll.

\section{Manuele Fiors Comic Fräulein Else}

Seit einigen Jahren ist in Deutschland ein regelrechter Comic-Boom zu verzeichnen. Die Zahl von Feuilletonartikeln über Comics und ihre Macher hat ebenso zugenommen wie Ausstellungen und Veröffentlichungen über Comics. Mehr und mehr beginnt sich auch die Literaturwissenschaft für dieses Phänomen zu interessieren, wobei neben komplexen und neuartigen Bild-Text-Kombinationen, die für Fragen der Intermedialitätsforschung besonders reichhaltiges Anschauungsmaterial bieten, insbesondere der Literatur-Comic in den Fokus des Interesses gerückt ist, wie Monika Schmitz-Emans in ihrem gleichnamigen Standardwerk belegt. ${ }^{29}$ Ähnlich wie bei Literaturverfilmungen und Drameninszenierungen handelt es sich bei Literatur-Comics

[...] um Rezeptionsprozesse, in deren Verlauf ein neues Werk entsteht. Abgesehen von jeweils eigenen Anspruch dieses neuen Werks auf die Wahrnehmung seiner Eigenart eröffnet es auch Perspektiven auf seine Vorlage: Es hebt an dieser das eine hervor, lässt das andere unberücksichtigt, setzt Akzente, verwandelt, verdeutlicht. ${ }^{30}$

Dieser immer häufiger zu beobachtende Bezug auf bekannte, kanonische Prätexte kann dabei als geschickte Profilierungs- oder auch Nobilitierungsstrategie verstanden werden, den Comic endgültig vom Verdacht der Primitivität und seiner rein unterhaltenden Funktion zu befreien und als ebenbürtige Kunstform zu etablieren.

\footnotetext{
${ }^{28}$ Vgl. dazu Alexandra Tacke: Schnitzlers „Fräulein Else“ und die Nackte Wahrheit. Novelle, Verfilmungen und Bearbeitungen. Köln/Weimar/Wien 2016.

${ }^{29}$ Vgl. dazu Monika Schmitz-Emans: Literatur-Comics. Adaptionen und Transformationen der Weltliteratur. Berlin 2012.

${ }^{30}$ Ebd., S. 11 [Kursive im Original].
} 
Neben dem Bezug auf literarische Klassiker kann auch ein künstlerischer Zeichenstil zur Nobilitierung des Ausdrucksmediums Comic beitragen. Der italienische Comic-Zeichner Manuele Fior gehört zu denjenigen Comiczeichnern, die für jeden Comic eine markante Zeichensprache entwickeln, gerne mit Farbe arbeiten und häufig Bezüge zur Kunstgeschichte herstellen. In seinem preisgekrönten und hoch gelobten Fräulein Else Comic (franz. 2009/dt. 2010) hat er sich für aquarellartige Zeichnungen entschieden, die sich durch eine spezielle Farbdramaturgie auszeichnen und Bezug auf die Wiener Moderne, insbesondere auf die Ästhetik von Gustav Klimt und Egon Schiele, nehmen. ${ }^{31}$ Die Bildästhetik der Wiener Moderne in $\mathrm{Zu}$ sammenhang mit Schnitzlers erzähltechnischer Neuerung des inneren Monologs zu bringen, macht den innovativen Kern des Fräulein Else Comics aus.

In den Rezensionen ist immer wieder die Referenz auf Egon Schieles Darstellungen von ausgemergelten und ausgezerrten Körpern hingewiesen worden. Auch in Fiors Farbdramaturgie, die von warmen Rot- und Gelbtönen über giftiges Grün bis hin zu kalten Grau-, Blau- und Schwarznuancen geht, hält er sich an die Farbskala der Wiener Moderne und findet Kolorierungen für Elses unterschiedliche Stimmungen. Dominieren am Anfang noch zarte Gelb-, Blau- und Rosétöne, nehmen die dunklen, eisigen und kalten Farben zum Schluss der Novelle immer mehr zu. Bemerkenswert ist darüber hinaus, wie Manuele Fior mit den Panelstrukturen und Rahmungen verfährt, indem er mit diesen spielt, sie auflöst, in Bruchstücke zerstückelt, in die Länge zieht, weitet oder diffus auslaufen lässt. Die Nutzung von zerflieBenden Aquarellfarben ist in dem Zusammenhang sicherlich als eine programmatische Entscheidung zu verstehen. Gerade in den Traumszenen zerfließen die Einzelbilder, verlieren ihre klaren Konturen.

Genuin filmische Einstellungen wie Panorama-Shot, Zoom oder Parallelmontage ermöglichen es wiederum, die Innenperspektive von Else wiederzugeben, ja werden geradezu exzessiv von Fior eingesetzt, um Elses Gespaltenheit zwischen ihrem Dasein als Beobachtende und Beobachtete umso markanter umzusetzen. Schon Torsten Hoffmann hat darauf hingewiesen, dass Fior es

[...] allein mit bildnerischen Mitteln [gelingt] die kategoriale Trennung zwischen Nullfokalisierung, interner und externer Fokalisierung aufzulösen. Zu diesem Zweck arbeitet er in der Körperdarstellung mit zwei unterschiedlichen Fokalisierungsstrategien [...]: erstens mit schnellen Fokalisierungswechseln, zweitens mit einer Fokalisierungsdiffusion. ${ }^{32}$

${ }^{31}$ Vgl. Manuele Fior: Fräulein Else nach der Novelle von Arthur Schnitzler. Text und Zeichnungen: Manuele Fior, Übersetzung, Textbearbeitung: Maximilian Lenz, Lettering: Tinet Emgren, Herausgeber: Johann Ulrich. Berlin: Avant Verlag 2010. (Zuvor 2009 im französischen Verlag (C) Guy Delcourt Productions erschienen). Der Comic gewann u. a. 2009 den Prix de la Ville de Genève.

${ }^{32}$ Torsten Hoffmann: ,Das nicht, bitte das nicht!' Körperdarstellungen in Comicversionen von Schnitzlers „Fräulein Else“ und Kafkas „Die Verwandlung“. In: Florian Trabert, Mara StuhlfauthTrabert, Johannes Waßmer (Hrsg.): Graphisches Erzählen. Neue Perspektiven auf Literaturcomics. Bielefeld 2015, S. 43-63, hier S. 46 [Kursive im Original]. 

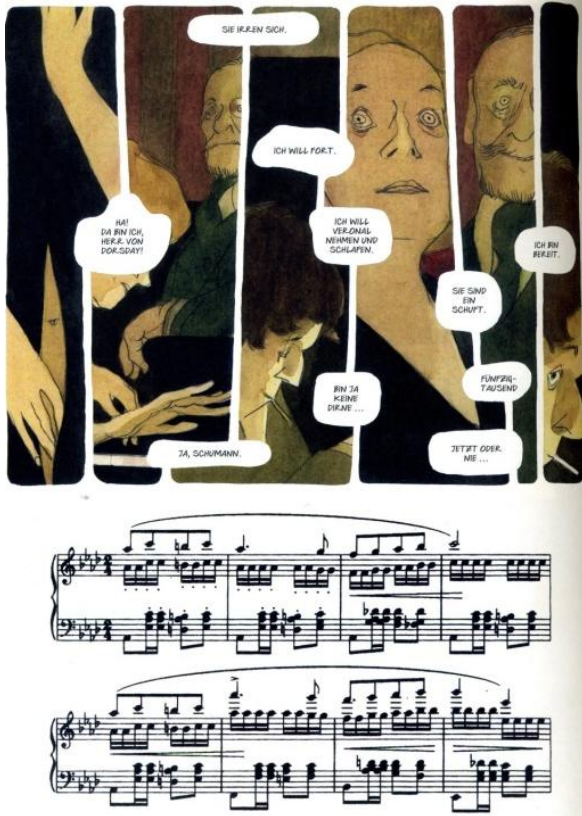

Abb. 4. (C) Manuele Fior und Éditions Delcourt

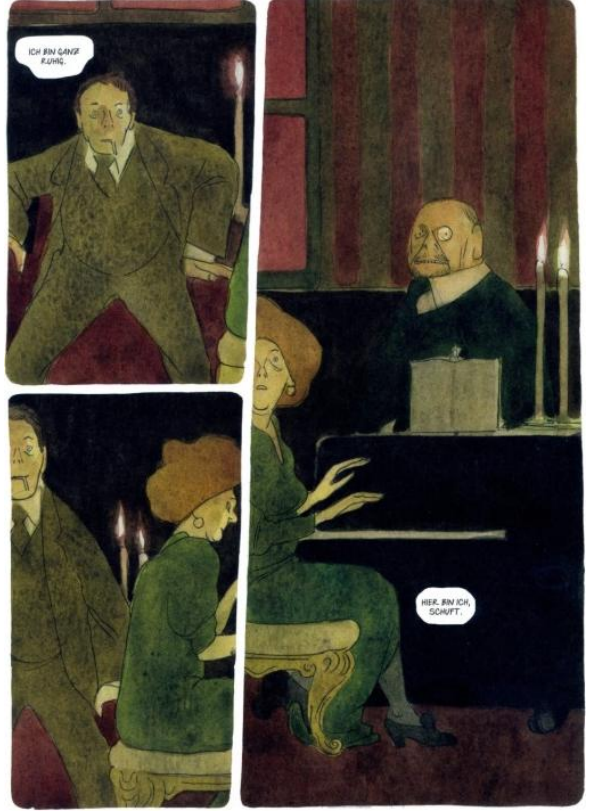

Abb. 5. (C) Manuele Fior und Éditions Delcourt

Ein besonders raffiniertes Spiel mit Rahmungen und Entrahmungen, Zerstückelungen und Zusammenfügungen betreibt Fior - wie auch Hoffmann bemerkt hat - in der Selbstentblößungsszene von Fräulein Else, die im Folgenden exemplarisch näher analysiert werden soll. Ähnlich wie Schnitzler auf syntaktischer Ebene zerhackt Fior den inneren Gedankenstrom von Else in Einzeläußerungen und -bilder. Die Sprechblasen mit Satzfetzen wirken wie Scharniere oder auch Pflaster, die die schwer erkennbaren, fragmentarischen Einzelbilder überhaupt zusammenhalten. Augen, Nase, Hände und ein Bauchnabel sind erkennbar, wenngleich sie schwer zuzuordnen sind, zumal auch die Blickperspektive permanent wechselt (Abb. 4). Mal ist Else die Blickende, dann wieder die Erblickte. Der Moment kurz vor der Entblößung wird bei Fior als ein Augenblick der körperlichen Dekomposition gezeigt, der die Subjektund Objektposition verschwimmen lässt und die Erzählkonventionen des Comics sprengt. Die Dekomposition steht im starken Kontrast zu der einheitlichen Komposition des Notenbildes, aber auch zu den klar strukturierten Bildern der gegenüberliegenden Seite (Abb. 5).

In die schockierten Blicke von Dorsday, der Klaviervirtuosin und dem Filou blickend, nimmt der Leser des Comics für einen kurzen Augenblick die Position der angeschauten Else ein - wird in ihre Lage versetzt, wird selbst zum Objekt des Blicks. Die Buchseite umwendend, erlangt der Leser scheinbar die Subjektposition wieder zurück. Denn entblößt und nackt steht Else ganz und in voller Pracht vor ihm (Abb. 6). 
$\mathrm{Zu}$ schamlos und direkt ist allerdings Elses Blick, als dass von einer sicheren Betrachterposition gesprochen werden kann. Darüber hinaus handelt es sich um ein Bildzitat, das die ambivalente Position zwischen Subjekt- und Objektsein zum Thema hat.

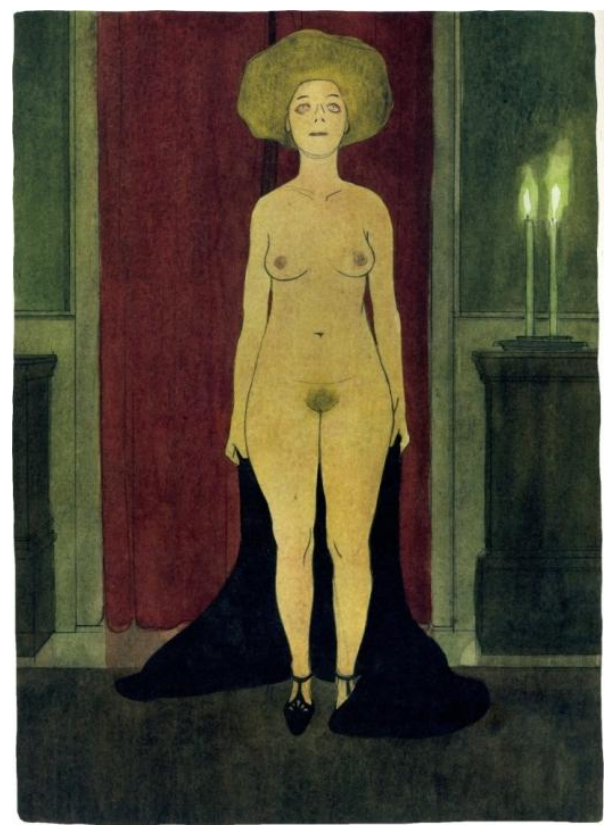

Abb. 6. (C) Manuele Fior und Éditions Delcourt
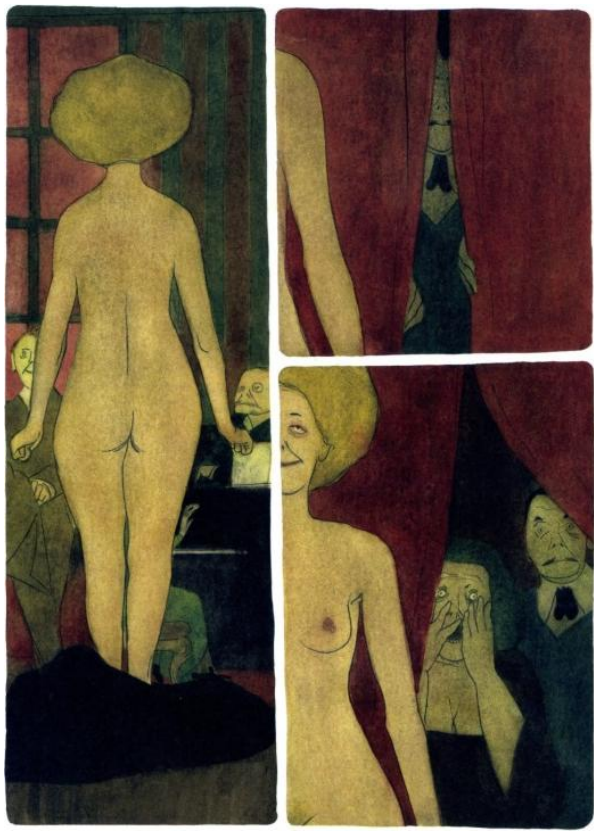

Abb. 8. (C) Manuele Fior und Éditions Delcourt

Wie unschwer zu erkennen ist, bezieht sich Manuele Fior auf Gustav Klimts berühmtes Gemälde Nuda Veritas aus dem Jahr 1899 (Abb. 7). Mit dem Bild hatte Klimt öffentlich Stellung gegen die Vorwürfe bezogen, die seine Fakultätsbilder ausgelöst hatten. Ebenso wie in den Fakultätsbildern geht es Klimt in seiner Version der Nuda Veritas, um eine Verschiebung der Möglichkeiten, Nacktheit darstellen zu dürfen. Als personifizierte Wahrheit hat Klimts Nuda Veritas ,alle historischen Verschleierungen abgelegt und schärft in ihrer Nacktheit den Blick für das Wesentliche. ${ }^{\text {“33 }}$ Auch wenn sie mit ihren offenen roten Haaren ebenso anstößig und verführerisch dargestellt ist wie Eva, hat Klimt den Sündenfall umgedeutet. Die Schlange beißt nicht mehr die Wade der Frau, sondern fokussiert stattdessen herausfordernd den Bildbetrachter. „Die Schlange ist überwunden, und Schuld findet

${ }^{33}$ Tobias G. Natter, Max Hollein (Hrsg.): Die nackte Wahrheit. Klimt, Schiele, Kokoschka und andere Skandale. München/Berlin/London/New York 2005, S. 108. 


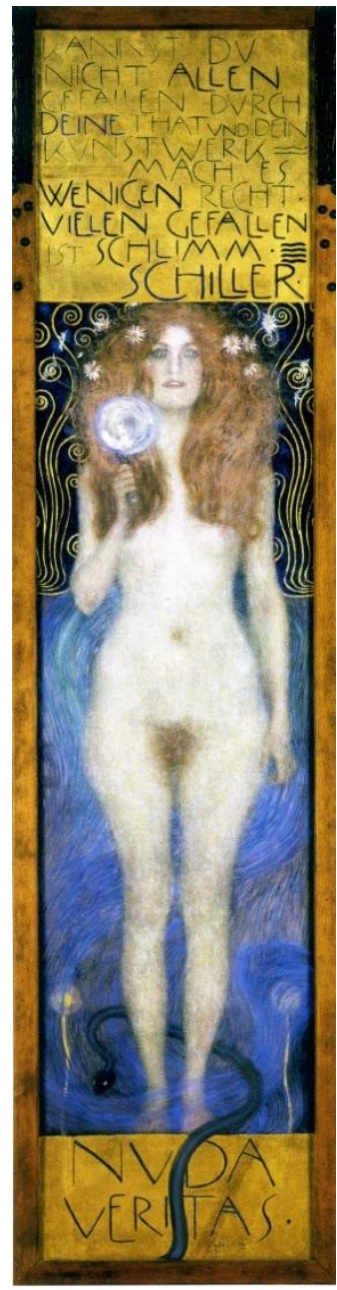

Abb. 7. Gustav Klimt: Nuda Veritas, 1899 Österreichisches Theatermuseum, Wien, Öl auf Leinwand, $252 \times 56,2 \mathrm{~cm}$, Nachlass Bahr-Mildenburg

sich nun nicht mehr auf Seiten der sich Darbietenden, son-

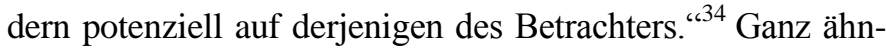
lich kann auch der Spiegel gedeutet werden, den die Nuda Veritas in ihrer rechten Hand hält und dem Bildbetrachter ostentativ entgegen- bzw. vorhält.

Indem Fior sich auf Klimts Gemälde bezieht, streicht er das Subversive an Elses Selbstentblößung hervor. Denn die Subjekt- und Objektposition gerät bei ihm - ebenso wie bei Klimt und auch in Schnitzlers Novelle - ins Wanken. Das wird noch deutlicher auf der nächsten Seite, wo der schamlose Blick auf die Nuda Veritas (von Dorsday und von der Gesellschaft) selbst in den Blick gerät. Nachdem der schwarze Mantel als erster Vorhang geöffnet worden ist und den Blick auf die Nuda Veritas freigegeben hat, öffnet sich ein weiterer Vorhang hinter dem Vorhang, der nun die schamlosen Betrachter selbst ausstellt, sie unwillentlich auf die Bühne zerrt und sie - nicht die Angeschaute - dadurch in den Blick nimmt. Das Hinter-der-Szene, das Obszöne, rückt in den Blick. Es sind verzerrte Blicke mit weit geöffneten Augen, die mehr Entsetzen als lustvolles Schauen offenbaren (Abb. 8).

Schließlich zerstört Elses hysterisches Gelächter den Anblick des stillgestellten, nackten Körpers. Konvulsivische Windungen und Bewegungen führen zum endgültigen Zusammenbruch des Nacktbildes, lassen ein hässliches Zerrbild entstehen und führen zunächst - anders als in der Novelle - zum völligen Verstummen von Elses Stimme (Abb. 9). Drei volle Seiten lang werden nur die Gespräche zwischen Paul, Cissy und der Tante wiedergegeben, die Else in ihrer Ohnmacht belauscht. Elses eigene Stimme scheint komplett verstummt. Erst allmählich kehrt sie als zarte Off-Stimme wieder zurück.

Schon zuvor war es vornehmlich der Rinnstein, das Zwischen-den-Panels, das Außerhalb der Einzelbilder, - dort auch, wo die Noteneinfügungen von Fior platziert werden - von dem aus Elses Stimme zu vernehmen gewesen ist. Fior betont dadurch die Wichtigkeit des Rinnsteins beim Comic, dessen Bedeutung schon Scott McCloud in seiner Comic-Definition hervorgehoben hat: Denn

\footnotetext{
${ }^{34}$ Ebd.
} 

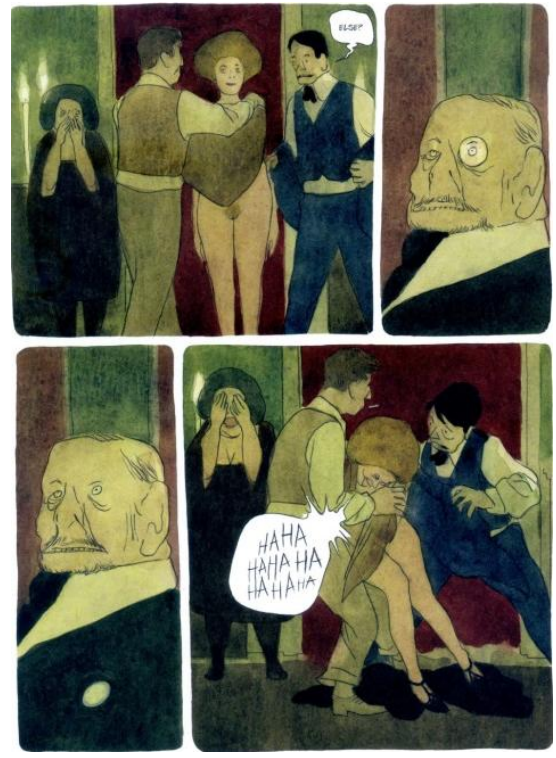

Abb. 9. (C) Manuele Fior und Éditions Delcourt
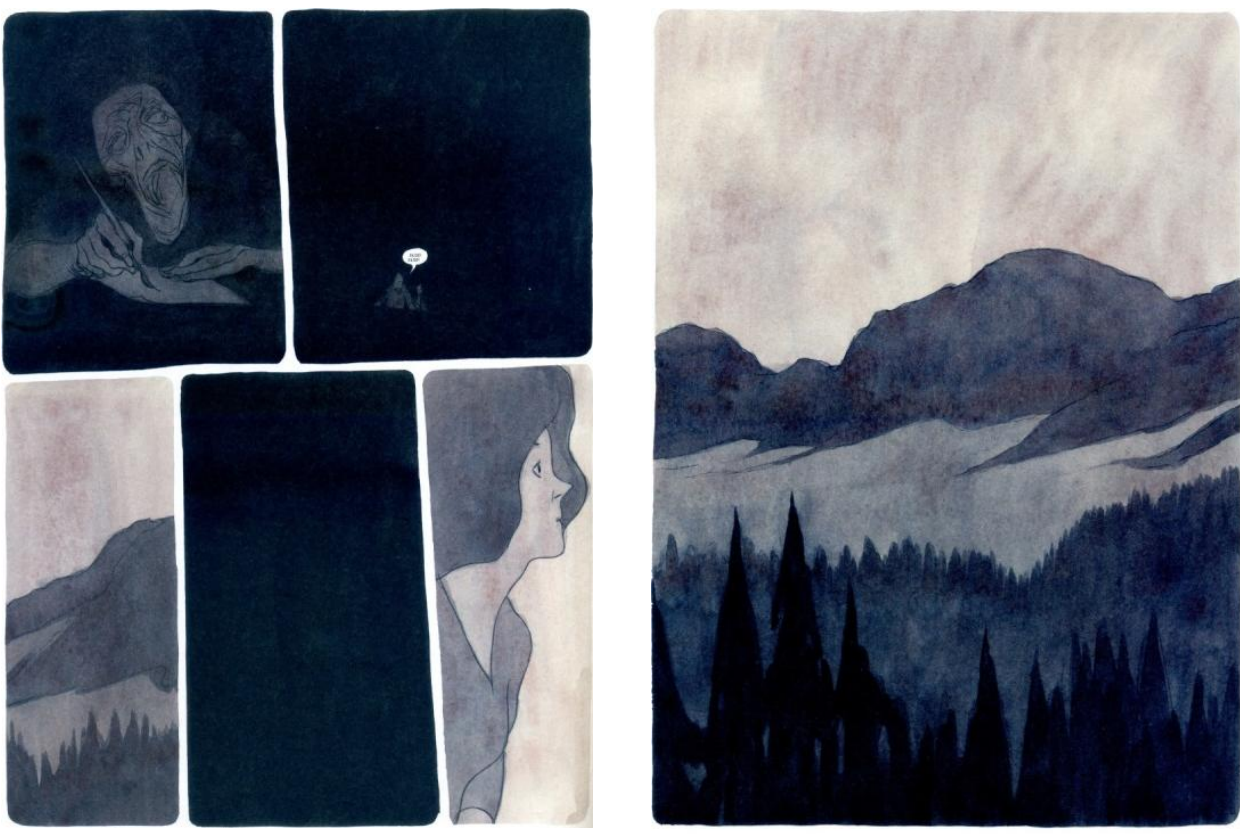

Abb. 10. (C) Manuele Fior und Éditions Delcourt

Abb. 11. (C) Manuele Fior und Éditions Delcourt 
Comics als Zeichensequenzen bestehen aus diskreten Elementen, die das Auge des Betrachters zu Kontexten zusammenliest. Entscheidend ist der Raum „dazwischen“, die Differenz zwischen den Zeichen. Im Comic wird er meist als die Fläche zwischen den Panels greifbar, die das Auge des Lesers kreativ überbrückt. ${ }^{35}$

Lassen einen die meisten Comics die Wichtigkeit des Rinnsteins vergessen, wird dieser bei Fior prominent hervorgehoben. Elses Stimme begnügt sich dabei nicht nur mit dem ihr zur Verfügung gestellten Raum zwischen den Bildern, sondern durchlöchert diese auch, legt sich über sie, zerfranst sie an ihren Rändern, knabbert sie an. Das Zwischen-den-Zeilen bzw. das Zwischen-den-Panels wird wichtig und verleiht jener unerhörten, ungehörten Stimme von Fräulein Else zweitweise einen Ort, bis diese am Ende - nachdem sie ein letztes Mal geäußert hat: „Ich will nicht sterben.“ - völlig verstummt und nur noch in einem düsteren Schwarzbild als AnwesendAbwesende visualisiert wird (Abb. 10). Elses Hilfeschrei bleibt bei Fior somit ein stummer, der höchstens in einem verschneiten Gebirgstal, wie es das letzte Panel des Comics zeigt, als kaum wahrnehmbares Echo nachzuklingen vermag (Abb. 11).

Fior rekurriert in seinem Comic damit weniger auf den Eurydike-Mythos - wie er am Ende der Novelle von Schnitzler eingewoben wird - als auf den Mythos von der griechischen Bergnymphe Echo, die keinen Körper mehr besitzt und nur noch Stimme ist. Ähnlich wie Else im inneren Monolog vorwiegend Sprachklischees ihrer Zeit wiederholt, kennt Echo auch keine eigene Rede, sondern nur eine reproduktive Sprache. Doch auch wenn Echos Stimme weniger Sinn produziert, macht sie sich am Ende als solche in ihrer Materialität umso nachhaltiger bemerkbar, indem sie die eigene anwesende-abwesende Präsenz behauptet - als leises, aber doch deutlich vernehmbares Echo, das sich wie die weiße Schneedecke über ein eisiges Gebirgstal legt.

\section{Literatur}

Allerkamp, Andrea: „Ich schreibe ja keine Memoiren“. Über die ars memoriae als Spiel zwischen Bild und Text in Schnitzlers „Fräulein Else“. In: Cahiers d'Etudes Germaniques. Heft 29/1995, S. $95-108$.

Brandstetter, Gabriele: Divested Interests. Ökonomie der Entblößung in Arthur Schnitzlers „Fräulein Else “ und Marina Abramovic' ,Freeing the Body“. In: Kerstin Gernig (Hrsg.): Nacktheit. Ästhetische Inszenierungen im Kulturvergleich. Köln/Weimar/Wien 2002, S. 241-271.

Diersch, Manfred: Empiriokritizismus und Impressionismus. Über Beziehungen zwischen Philosophie, Ästhetik und Literatur um 1900 in Wien. Berlin 1977.

${ }^{35}$ Scott McClouds Comicdefinition in seinem Comic Understanding Comics (1993/94) hat Monika Schmitz-Emans prägnant zusammengefasst - Schmitz-Emans: Literatur-Comics, S. 48f. 
Eilert, Heide: Das Kunstzitat in der erzählenden Dichtung. Studien zur Literatur um 1900. Stuttgart 1991, S. 323-335.

Fior, Manuele: Fräulein Else nach der Novelle von Arthur Schnitzler. Text und Zeichnungen: Manuele Fior, Übersetzung, Textbearbeitung: Maximilian Lenz, Lettering: Tinet Elmgren, Herausgeber: Johann Ulrich. Berlin 2010.

Fliedl, Konstanze: Arthur Schnitzler. Stuttgart 2005.

Gomes, Mario: Gedankenlesemaschinen. Modelle für eine Poetologie des Inneren Monologs. Freiburg i.Br./Berlin/Wien 2008.

Hoehnisch, Erika: Das gefangene Ich. Studien zum inneren Monolog in modernen französischen Romanen. Heidelberg 1967.

Hoffmann, Torsten: ,Das nicht, bitte das nicht! ' Körperdarstellungen in Comicversionen von Schnitzlers „Fräulein Else“ und Kafkas ,Die Verwandlung. “ In: Florian Trabert, Mara Stuhlfauth-Trabert, Johannes Waßmer (Hrsg.): Graphisches Erzählen. Neue Perspektiven auf Literaturcomics. Bielefeld 2015, S. 43-63.

Huber, Martin: Optische Musikzitate als Psychogramm in Arthur Schnitzlers „Fräulein Else“. In: ders.: Text und Musik. Musikalische Zeichen im narrativen und ideologischen Funktionszusammenhang ausgewählter Erzähltexte des 20. Jahrhunderts. Frankfurt a. M./Berlin/Bern/New York/Paris/Wien 1991, S. 78-91.

Krényi, Karl: Die Mythologie der Griechen - Die Götter- und Menschheitsgeschichten. München 1994.

Lange-Kirchheim, Astrid: Die Hysterikerin und ihr Autor. Arthur Schnitzlers Novelle „Fräulein Else“ im Kontext von Freuds Schriften zur Hysterie. In: Thomas Anz (Hrsg.): Psychoanalyse in der modernen Literatur. Kooperation und Konkurrenz. Würzburg 1999, S. 111-134.

Morris, Craig: Der vollständige innere Monolog: eine erzählerlose Erzählung? Eine Untersuchung am Beispiel von „Leutnant Gustl“ und „Fräulein Else“. In: Modern Austrian Literature. Journal of the International Arthur Schnitzler Research Association 31, Nr. 2/1998, S. 30-51.

Natter, Tobias G.; Hollein, Max (Hrsg.): Die nackte Wahrheit. Klimt, Schiele, Kokoschka und andere Skandale. München/Berlin/London/New York 2005.

Polt-Heinzl, Evelyne: Erläuterungen und Dokumente. Arthur Schnitzler „Fräulein Else“. Stuttgart 2002, S. 54-55.

Pross, Caroline: Näherungswerte. Theatralik des Wirklichen und realistisches Erzählen bei Arthur Schnitzler. In: Ethel Matala de Mazza, Clemens Pornschlegel (Hrsg.): Inszenierte Welt. Theatralität als Argument literarischer Texte. Freiburg i.Br. 2003, S. 227-249.

Prutti, Brigitte: Weibliche Subjektivität und das Versagen des sanften Patriarchen in Schnitzlers „Fräulein Else“. In: Orbis Litterarum Nr. 59/2004, S. 159-187.

Salten, Felix: ,Fräulein Else“. In: Neue Freie Presse. Morgenblatt. Nr. 21623. Wien 1924, S. 1-3.

Scheffel, Michael: Nachwort. In: Arthur Schnitzler: Spiel im Morgengrauen. Erzählungen 1923-1931. Mit einem Nachwort von Michael Scheffel. Ausgewählte Werke in acht Bänden. Hrsg. von Heinz Ludwig Arnold, Bd. 3. Frankfurt a. M. 1999, S. 385-394.

Schmidt-Dengler, Wendelin: Inflation der Werte und Gefühle. Zu Arthur Schnitzlers „Fräulein Else“. In: Giuseppe Farese (Hrsg.): Akten des Internationalen Symposiums „Arthur Schnitzler und seine Zeit“. Bern 1985, S. 170-181.

Schmitz-Emans, Monika: Literatur-Comics. Adaptionen und Transformationen der Weltliteratur. Berlin 2012.

Schneider, Gerd K.: Ton- und Schriftsprache in Schnitzlers „Fräulein Else“ und Schumanns „Carnaval ". In: Modern Austrian Literature. Journal of the International Arthur Schnitzler Research Association. Vol. 2, Nr. 3/1969, S. 17-20. 
Schnitzler, Arthur: Fräulein Else. In: ders.: Spiel im Morgengrauen. Erzählungen 1923-1931. Mit einem Nachwort von Michael Scheffel. Ausgewählte Werke in acht Bänden. Hrsg. von Heinz Ludwig Arnold, Bd. 3. Frankfurt a. M. 1999, S. 7-73.

Tacke, Alexandra: Schnitzlers „Fräulein Else“ und die Nackte Wahrheit. Novelle, Verfilmungen und Bearbeitungen. Köln/Weimar/Wien 2016.

Urbach, Reinhard: Schnitzler-Kommentar zu den erzählenden Schriften und dramatischen Werken. München 1974, S. 130-132.

Zenke, Jürgen: Die deutsche Monologerzählung im 20. Jahrhundert. Köln/Wien 1976. 